\title{
Null Stellar Intensity Interferometry
}

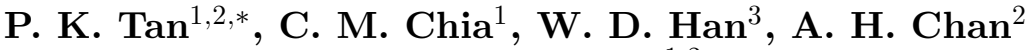 \\ and C. Kurtsiefer ${ }^{1,2}$ \\ ${ }^{1}$ Centre for Quantum Technologies, Singapore 117543 \\ ${ }^{2}$ Dept. of Physics, National University of Singapore, Singapore 117551 \\ ${ }^{3}$ Dept. of Electrical \& Computer Engineering, NUS, Singapore 117583 \\ *email: pengkian@physics.org
}

\begin{abstract}
Since the discovery of the first exoplanet in 1989, though over 850 candidates have been verified (Schneider 2012), few are similar to our Earth in terms of mass and size. Hence here we would like to propose the revival and improvement of optical intensity interferometry to achieve sub-milliarcsecond resolution, which promises also to be less sensitive to weather conditions, light pollution and optomechanical alignments, yet only requiring baselines $<100 \mathrm{~m}$.
\end{abstract}

Keywords. exoplanet, g2, intensity interferometry, photon bunching, second order correlation

\section{Introduction}

Thermal light sources such as stars exhibit photon bunching, as demonstrated by Hanbury Brown and Twiss (1956), but has been a neglected feature in astronomy till recently (Hyland 2005, Ofir et al. 2006, Capraro et al. 2009, Naletto et al. 2009, Dravins et al. 2012). A reason for this is due to stellar blackbody radiation having a typical coherent timescale of only femtoseconds, and existing detectors are simply not fast enough to resolve explicitly, thus requiring much larger telescope apertures or arrays to compensate for the signal-to-noise ratio (SNR). We propose an alternative solution by filtering the starlight down to the regime of nanosecond coherence, and so allowing for the direct precision measurements of $\mathrm{g}^{(2)}(\tau)$, and consequently spatial $\mathrm{g}^{(2)}$.

\section{Theory}

Assuming a planar light source that is spatially incoherent and quasi-monochromatic, the far-field form of the Van Cittert Zernike theorem states that the equal-time complex degree of coherence at two points in the generated field is equal to the Fourier transform of the intensity distribution of the source (Mandel et al. 1995, Foellmi 2009):

$$
g^{(1)}\left(\vec{r}_{\alpha}, \vec{r}_{\beta}\right)=\frac{e^{i \bar{k}\left(\vec{r}_{\alpha}-\vec{r}_{\beta}\right)} \int_{\vec{r}_{s}} I\left(\vec{r}_{s}\right) e^{i \bar{k}\left(\hat{r}_{\alpha}-\hat{r}_{\beta}\right) \cdot \vec{r}_{s}} d \vec{r}_{s}}{\int_{\vec{r}_{s}} I\left(\vec{r}_{s}\right) d \vec{r}_{s}}
$$

On further assuming the light source, being a star, to have a circular aperture and of uniform intensity (neglecting limb darkening), the relation simplifies to:

$$
g^{(1)}\left(\vec{r}_{\alpha}, \vec{r}_{\beta}\right)=\frac{2 J_{1}(\nu)}{\nu}
$$

where $J_{1}$ is the Bessel function of the first kind in the first order, and $\nu$ is the spatial frequency. In the case of a thermal light source, we can then thus extract this spatial coherence information from the second order correlation via the relation:

$$
g^{(2)}=1+\left|g^{(1)}\right|^{2}
$$




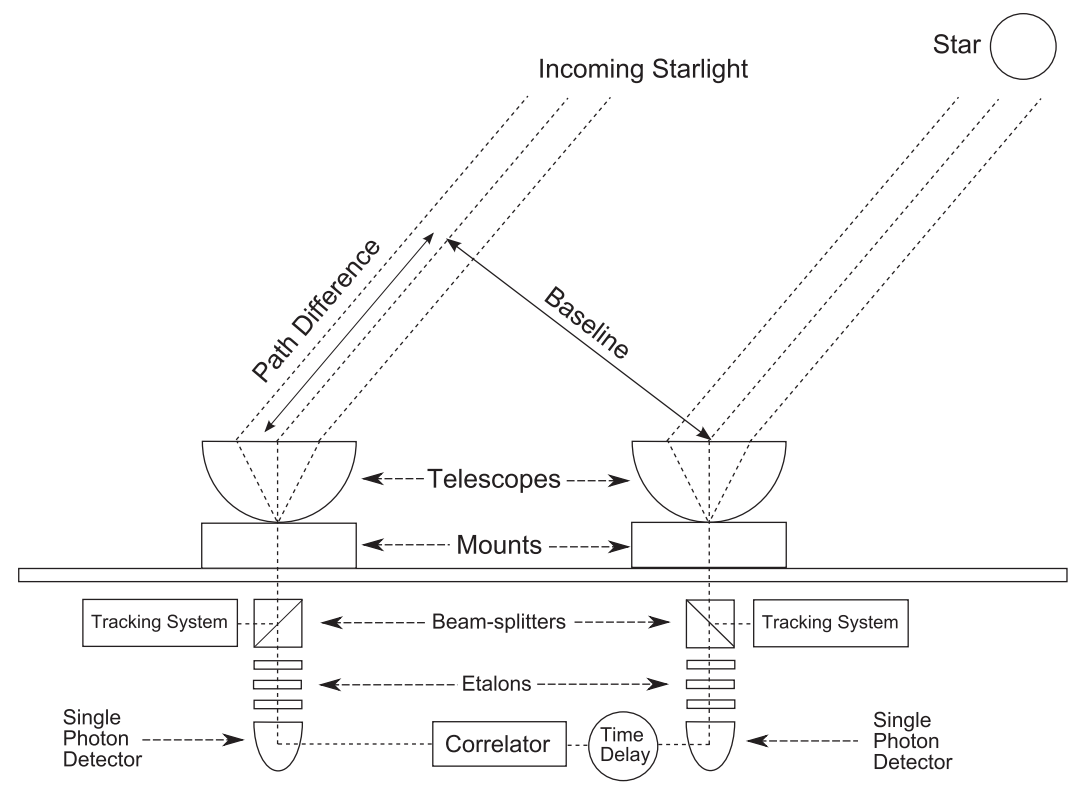

Figure 1. Experimental Schematic Overview

\section{Measurements}

We measure $g^{(2)}$ using two photon detectors at positions $\vec{r}_{\alpha}$ and $\vec{r}_{\beta}$, where $\mathrm{b}$ is the baseline, $N_{\alpha \beta}$ is the number of coincidence counts, $N_{\alpha, \beta}$ are the number of individual counts, $\Delta t$ is the coincidence window and $\Delta T$ is the total observation window:

$$
g^{(2)}(b)=\frac{N_{\alpha \beta}}{N_{\alpha} N_{\beta}} \frac{\Delta T}{\Delta t}
$$

with a useful result of:

$$
d_{s}=1.22 \frac{\bar{\lambda}}{b_{0}}
$$

where $d_{s}$ is the stellar angular diameter, $\bar{\lambda}$ is the mean wavelength observed and $b_{0}$ being the baseline at the first $g^{(2)}$ minima. As planets are much smaller and non-luminous, we hereby propose a novel new method of indirect detection: whenever a planet passes through the line of sight between us and its host star, thus partially nulling the star, we would expect the complex degree of coherence to change accordingly:

$$
g_{\text {transit }}^{(1)}=\frac{\mathcal{F}\left\{I_{s}(\vec{r})-I_{p}(\vec{r})\right\}}{\int_{\vec{r}}\left[I_{s}(\vec{r})-I_{p}(\vec{r})\right] d \vec{r}}=\frac{\mathcal{F}\left\{I_{s}(\vec{r})\right\}-\mathcal{F}\left\{I_{p}(\vec{r})\right\}}{\int_{\vec{r}} I_{s}(\vec{r}) d \vec{r}-\int_{\vec{r}} I_{p}(\vec{r}) d \vec{r}}
$$

which we can extract from its associated $\mathrm{g}^{(2)}(\mathrm{b})$ and thus detecting both the transit event and deducing the planetary angular diameter $\mathrm{d}_{p}$ via:

$$
g_{\text {transit }}^{(2)}(b)=1+\left|\frac{2 J_{1}\left(\frac{\pi d_{s}}{\bar{\lambda}} b\right)-\left(\frac{d_{p}}{d_{s}}\right) 2 J_{1}\left(\frac{\pi d_{p}}{\bar{\lambda}} b\right)}{\frac{\pi d_{s}}{\bar{\lambda}} b\left(1-\left(\frac{d_{p}}{d_{s}}\right)^{2}\right)}\right|^{2}
$$

For illustration, we would expect an observation like Figure 2, for the case of a Jupiterlike planet orbiting around Sirius, which is 8.6 light-years away and roughly 2.9 times larger than our Sun, measuring at $\bar{\lambda}=400 \mathrm{~nm}$. The first minima is at $\mathrm{b}=16.9 \mathrm{~m}$, corresponding to an angular diameter of 5.9 milliarcseconds for Sirius. 


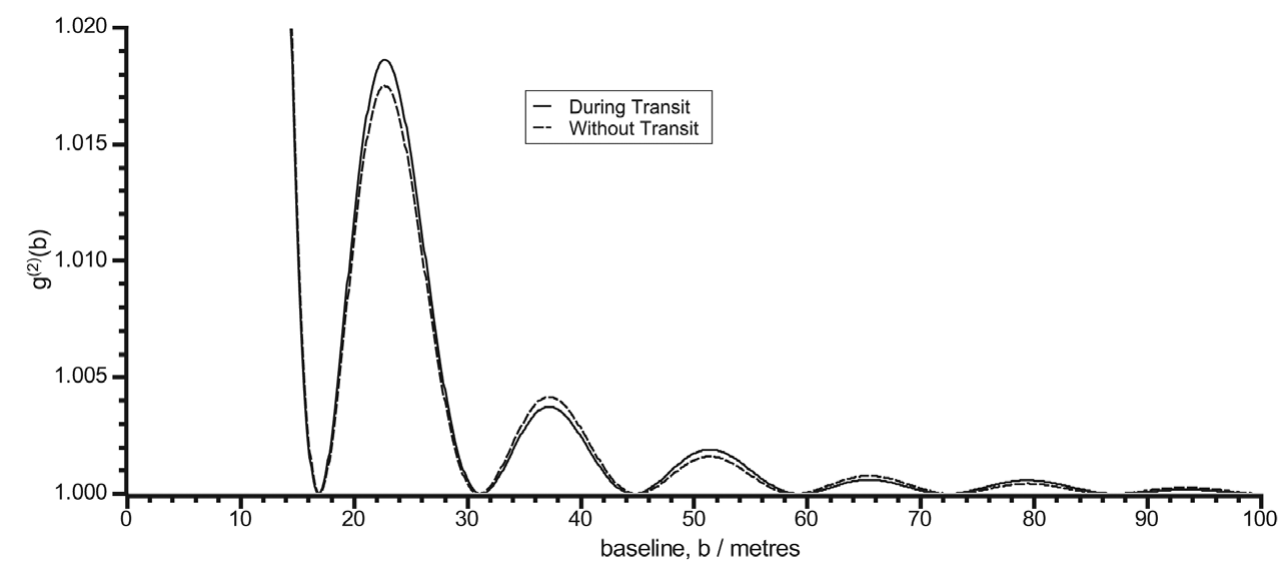

Figure 2. Hypothetical Exoplanetary Transit

For a planetary transit, we would ideally expect to measure a $\Delta g^{(2)}$ of 0.001 in the peak at the baseline of $22.7 \mathrm{~m}$, assuming that the telescope aperture and integration time is sufficient to overcome poissonian noise to resolve this difference. Also neglected is the limb darkening coefficient, which can be corrected by measuring subsequent peaks and performing reverse fourier transform to deduce the stellar spatial intensity profile. Instrumental errors such as detector quantum efficiencies, optical losses, starlight intensity variability should not contribute as they affect both coincidences and singles counts equally and thus cancel out. The alternating oscillating peaks is an useful signature.

\section{Temporal Coherence}

Due to the discreteness of spacetime, it has been predicted that there should be some degree of phase decoherence in photons from cosmic sources (Lieu et al. 2003, $\mathrm{Ng}$ et al. 2003, Maziashvili 2009), and that if accumulated over sufficient distances, might produce a measurable effect in its temporal coherence. Although filtering does not directly affect the SNR $=\frac{1}{2} \tau_{0}\left|g^{(1)}(\tau)\right|^{2}\left(\frac{1}{2} N_{\alpha} N_{\beta} \frac{\Delta T}{\Delta t}\right)^{\frac{1}{2}}$ as given by Capraro (2009); but our setup with a coherent timescale $\tau_{0}$ of $\approx 3 \mathrm{~ns}$ and actively quenched avalanche photon detectors (APDs) with $<40$ ps resolution should allow us to explicitly resolve the photon bunching events with $\approx 100$ timebins and thus derive more precise $g^{(2)}(\tau=0)$ values.

\section{References}

Brown, R. H. \& Twiss, R. Q. 1956, Nature, 178, 1046

Capraro, I., Naletto, G., Barbieri, C., Occhipinti, T., Verroi, E., Zoccarato, P., \& Di Paolo, A. 2009, Proc. of Science, QQ09, 012

Dravins, D., LeBohec, S., Jensen H., \& Nunez, P. D. 2012, New Astron. Revs, 56, 143

Foellmi, C. 2009, A\&A, 507, 3

Hyland, D. 2005, Proc. of SPIE, 5905, 590511-1

Lieu, R. \& Hillman, L. W. 2003, ApJ (Letters), 585, L77

Mandel, L. \& Wolf, E. 1995, Optical Coherence \& Quantum Optics (Cambridge University Press)

Maziashvili, M. 2009, Astroparticle Physics, 31, 344

Naletto, G., et al. 2009, A\&GA, 508, 531

Ng, Y. J., Christianensen, W. A., \& Dam, H. V. 2003, ApJ (Letters), 591, L87

Ofir, A. \& Ribak, E. N. 2006, Proc. of SPIE, 6268, 62683S

Schneider, J. 2012, The Extrasolar Planets Encyclopaedia (http://exoplanet.eu/) 